\title{
Origin of large negative electrocaloric effect in antiferroelectric $\mathrm{PbZrO}_{3}$
}

\author{
Pablo Vales-Castro $\odot,{ }^{1, *}$ Romain Faye, ${ }^{2}$ Miquel Vellvehi $\odot,{ }^{3}$ Youri Nouchokgwe,,${ }^{2,4}$ Xavier Perpiñà $\odot,{ }^{3}$ J. M. Caicedo, ${ }^{1}$ \\ Xavier Jordà, ${ }^{3}$ Krystian Roleder, ${ }^{5}$ Dariusz Kajewski, ${ }^{5}$ Amador Perez-Tomas, ${ }^{1}$ Emmanuel Defay $\odot,{ }^{2}$ and Gustau Catalan ${ }^{1,6, \dagger}$ \\ ${ }^{1}$ Catalan Institute of Nanoscience and Nanotechnology (ICN2), Campus Universitat Autonoma de Barcelona, Bellaterra 08193, Spain \\ ${ }^{2}$ Materials Research and Technology Department, Luxembourg Institute of Science and Technology, rue du Brill 41, \\ Belvaux L-4422, Luxembourg \\ ${ }^{3}$ Institut de Microelectrònica de Barcelona (IMB-CNM,CSIC) Campus Universitat Autònoma de Barcelona (UAB), \\ Cerdanyola del Vallès, Barcelona 08193, Spain \\ ${ }^{4}$ University of Luxembourg, 2 avenue de l'Université, L-4365 Esch-sur-Alzette, Luxembourg \\ ${ }^{5}$ Institute of Physics, University of Silesia in Katowice, ul. Uniwersytecka 4, 40-00 Katowice, Poland \\ ${ }^{6}$ Institut Català de Recerca i Estudis Avançats (ICREA), Barcelona 08010, Catalunya
}

(Received 16 August 2020; revised 22 January 2021; accepted 2 February 2021; published 22 February 2021)

\begin{abstract}
We have studied the electrocaloric response of the archetypal antiferroelectric $\mathrm{PbZrO}_{3}$ as a function of voltage and temperature in the vicinity of its antiferroelectric-paraelectric phase transition. Large electrocaloric effects of opposite signs, ranging from an electrocooling of $-3.5 \mathrm{~K}$ to an electroheating of $+5.5 \mathrm{~K}$, were directly measured with an infrared camera. We show by calorimetric and electromechanical measurements that the large negative electrocaloric effect comes from an endothermic antiferroelectric-ferroelectric switching, in contrast to dipole destabilization of the antiparallel lattice, previously proposed as an explanation for the negative electrocaloric effect of antiferroelectrics.
\end{abstract}

DOI: 10.1103/PhysRevB.103.054112

\section{INTRODUCTION}

The electrocaloric effect (ECE) is the reversible temperature change $(\Delta T)$ of a material when a voltage step is applied or removed adiabatically [1]. It was first theorized in 1878 by Thomson [2] as the inverse of the pyroelectric effect, but it took 50 years until the ECE was first observed in ferroelectric Rochelle salt [3], and it was first quantitatively measured even later, by Hautzenlaub in 1943 [4]. Although initially it did not attract much attention because of the low-temperature increments achieved, a large EC temperature change was calculated in 2006 for ferroelectric thin films [5], prompting a surge of interest in this effect. The ECE is attractive as a way to develop solid-state cooling systems, and also because the theoretical efficiency goes up to $70 \%$, much higher than thermoelectrics $(10 \%)$ or even a conventional gas-cooling cycle (50\%) [1]. Moreover, it has a great potential for scalability, useful to cool down advanced integrated circuits or complex systems in ever more powerful chips and heating-prone computers, including emerging wide band-gap technologies (e.g., SiC, $\mathrm{GaN}$, or $\mathrm{Ga}_{2} \mathrm{O}_{3}$ ) that can operate at larger temperatures than the $175{ }^{\circ} \mathrm{C}$ limit of silicon devices [6]. The scalability of the electrocaloric effect comes from the fact that the large electric fields required to produce large temperature changes can be achieved with modest voltages in thin films, thanks to their reduced thickness and increased breakdown strength [5].

Antiferroelectrics (AFEs) are materials with antiparallel sublattices of electric dipoles that can be switched under electric field into a polar state. Their electrocaloric properties

\footnotetext{
*pablo.vales@icn2.cat

†gustau.catalan@icn2.cat
}

have been less investigated than those of ferroelectrics, but their study has increased since 2011 with the discovery of the anomalous electrocaloric effect [7] (also called negative electrocaloric effect), whereby applying a voltage causes a decrease in temperature $(\Delta T<0)$, rather than an increase. This "electrocooling" is contrary to the normal (or positive) ECE displayed by conventional ferroelectrics, which increase their temperature $(\Delta T>0)$ when the field is applied. This electrocaloric temperature change $\Delta \mathrm{T}$ can be extracted from Maxwell relations as defined by Eq. (1) (see Supplemental Material [8] and Refs. [9-13]). The interest in this anomalous effect was further enhanced by the report of indirectly measured "giant" negative electrocaloric effects in antiferroelectric thin films $[10,14]$.

$$
\Delta T=-\int_{E_{1}}^{E_{2}} \frac{T}{C_{E}(T, E)}\left(\frac{\partial D}{\partial T}\right)_{E} d E .
$$

\section{MICROSCOPIC ELECTROCALORIC MODELS}

It may seem surprising that a material can get colder when energy (voltage, in this case) is added to it. While macroscopically the negative electrocaloric effect occurs because $\left(\frac{\partial D}{\partial T}\right)_{E}>0$ [Eq. (1)], the microscopic mechanism that enables such behavior in antiferroelectrics is still being debated, and there are at least two possible explanations put forward in the community. On the one hand, when an electric field is adiabatically applied to an antiferroelectric, it destabilizes the dipole sublattice that is antiparallel to the applied field, as explained by Geng et al. [10,14] thus increasing its dipolar entropy $\left(S_{\text {dip }}\right)$ and thereby reducing its temperature (by decreasing the 

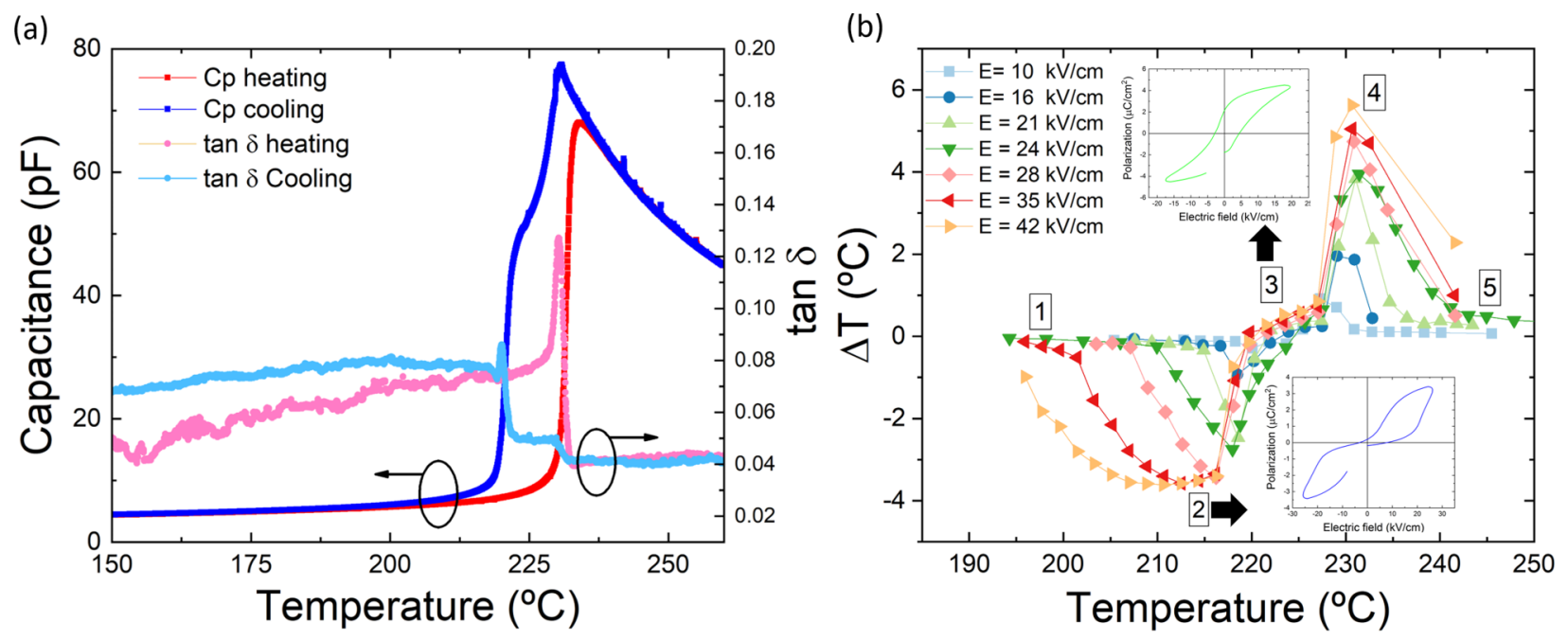

FIG. 1. (a) Capacitance and losses of $\mathrm{PbZrO}_{3}$ ceramics and (b) electrocaloric temperature change vs temperature of $\mathrm{PbZrO}_{3}$, characterized with an IR camera. Insets represent the polarization versus electric field hysteresis loops.

phononic contribution $S_{p h}$ ) to satisfy Eq. (1).

$$
\Delta S=\Delta S_{\text {dip }}+\Delta S_{p h}=0
$$

This is opposite to paraelectrics and ferroelectrics, where electric fields increase dipole alignment and thus reduce dipolar entropy (although, negative ECE can also appear in ferroelectrics when the polarization is antiparallel or not collinear with the applied electric field [15]).

On the other hand, a negative electrocaloric effect can also appear in any material that undergoes a field-induced first-order phase transition between two phases (provided the phase transition is endothermic), as already reported for some ferroelectrics [15-17] and also for antiferroelectric $\mathrm{PbZrO}_{3}$ [18]. This can take place as long as the field-induced phase transition is defined by a positive latent heat $L_{H}$ (defined by $\left.\Delta S_{L H}\right)$ and as long as this is larger than the entropy variation of the smooth and continuous change of $P$ through the transition $\left(\Delta S_{\tilde{P}}\right)$, according to Eq. (2) [19]. Note that Eq. (2) is just a generalization of Eq. (1) when latent heat has a role in the transition.

$$
\Delta S=\Delta S_{\text {dip }}+\Delta S_{p h}=\left(\Delta S_{\tilde{P}}+\Delta S_{L H}\right)+\Delta S_{p h}=0 .
$$

In this framework, the negative ECE in the prototypical antiferroelectric $\mathrm{PbZrO}_{3}$ would be best described not so much as a destabilization of a polar sublattice, but as a field-induced endothermic phase transition.

These two models predict rather different functionalities. In the sublattice destabilization ("dipole canting") model, once the antiparallel sublattice is switched, its entropy should decrease again as the dipoles have been realigned parallel to the field. The cooling achieved during the subcoercive part, i.e., below the antiferroelectric-ferroelectric transition field $\left(E_{\mathrm{AFE}-\mathrm{FE}}\right)$, should therefore turn to heating upon switching $\left(E>E_{\mathrm{AFE}-\mathrm{FE}}\right)$, and the net thermal balance after saturation would tend to be neutral or positive [10]. By contrast, if the negative electrocaloric effect relies on latent heat of transformation, cooling will be expected to increase, rather than decrease, when there is antiferroelectric switching until it saturates. Put another way: while the dipole-canting model relies on subswitching fields $\left(E<E_{\mathrm{AFE}-\mathrm{FE}}\right)$, the phasechange model requires overcoming the phase transition point $\left(E<E_{\mathrm{AFE}-\mathrm{FE}}\right)$. Clarifying which is the dominant contribution to the negative ECE of antiferroelectrics is therefore not only a fundamental science question; it is also essential for maximizing the response of electrocaloric devices as a function of voltage. Determining which of the two models (dipole canting [14] or latent heat of transformation [18]) dictates the large negative electrocaloric response of the archetypal antiferroelectric $\left(\mathrm{PbZrO}_{3}\right)$ is the aim of this investigation.

\section{RESULTS}

\section{A. Dielectric and electrocaloric measurements}

Antiferroelectric $\mathrm{PbZrO}_{3}$ ceramics were fabricated as reported in Ref. [20]. Their capacitance and losses as a function of temperature are shown in Fig. 1(a). On heating, there is a single peak at the Curie temperature $\left(T_{c}\right)$, signaling the transition from the antiferroelectric (orthorhombic, Pbam symmetry [21]) phase to the paraelectric (simple cubic, $P m 3$ $m$ symmetry [21]) one. On cooling, there is an additional shoulder at lower temperatures, suggesting the existence of a ferroelectric (rhombohedral, $R 3 c$ or $R 3 m$ symmetry [22]) stable intermediate phase. For the sake of simplicity, we concentrate the discussion on the electrocaloric response on heating, without loss of generality; the full set of results for heating and cooling are provided in Supplemental Material [8] (Section S1).

The electrocaloric response as a function of temperature and field is shown in Fig. 1(b), together with the relevant polarization versus field loops at different regions. We have labeled the ranges where qualitatively and quantitatively different behaviors are displayed. At low temperatures and/or with low voltages (range 1), there is only a small negative ECE $(\Delta T \leqslant-0.6 \mathrm{~K})$. Above a temperature-dependent critical field, there is a jump in the negative response (range 2), reaching a maximum temperature change of $\Delta T=-3.6 \mathrm{~K}$ for fields $\geqslant 35 \mathrm{kV} \mathrm{cm}^{-1}$. The maximum negative electrocaloric strength is very high, peaking at $\left(|\Delta T||\Delta E|^{-1}\right)_{\text {negECE }}=$ 
(a)

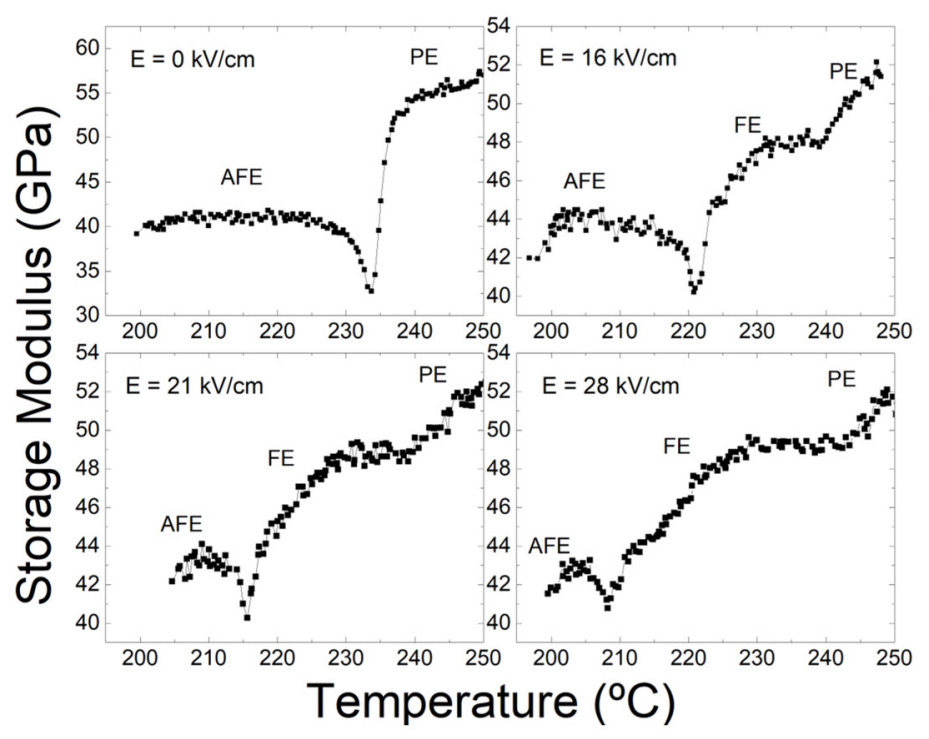

(b)

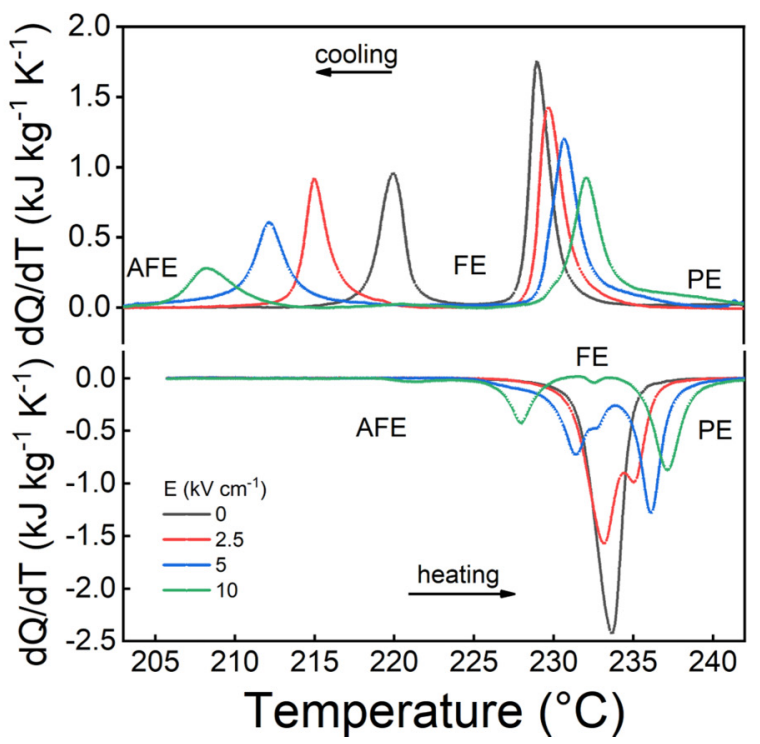

FIG. 2. (a) Storage modulus of a PZO ceramic vs temperature obtained with DMA. The discontinuities represent switching between different phases of PZO. (b) DSC measurements of a $\mathrm{PbZrO}_{3}$ ceramic at different electric fields for heating and cooling

$0.12 \mathrm{~K} \mathrm{~cm} \mathrm{kV}^{-1}$. At higher temperatures, the effect abruptly changes from large negative to almost zero or weakly positive in the temperature range between $\sim 220$ and $\sim 227{ }^{\circ} \mathrm{C}$ (range 3 ). Then there is another sharp increase, whereby the ECE abruptly rises to a positive peak (range 4) before dropping again to weakly positive (almost zero) above $T_{C}$ (range 5). The maximum positive ECE in range 4 was $\Delta T=+5.6 \mathrm{~K}$, and the maximum electrocaloric strength $\left(|\Delta T||\Delta E|^{-1}\right)_{\text {posECE }}=$ $0.18 \mathrm{~K} \mathrm{~m} \mathrm{kV}^{-1}$. The overall response in the whole temperature span (i.e., regions 1 through 5) is qualitatively similar to that predicted for Ba-doped $\mathrm{PbZrO}_{3}$ ceramics [23], for which doping stabilizes the FE intermediate phase over a wider temperature range.

The electrocaloric maxima (both negative and positive) are listed in Table S1 (Supplemental Material [8] and Refs. [9,18,23-41]) and are compared to other directly measured values reported in the literature. The maximum negative electrocaloric effect in $\mathrm{PbZrO}_{3}$ is significantly higher than any result previously reported by direct measurements. (Parenthetically, the positive electrocaloric peak is also among the highest, comparable to $\mathrm{PbSc}_{0.5} \mathrm{Ta}_{0.5} \mathrm{O}_{3}$ multilayer capacitors [40]). This high electrocaloric performance of $\mathrm{PbZrO}_{3}$ is intrinsic and not achieved through compositional finetuning: $\mathrm{PbZrO}_{3}$ is a pure compound and the antiferroelectric archetype.

\section{B. Mechanical analysis and calorimetry}

In order to determine the apparent link between electrocaloric response and field-induced phase transition, we have performed two additional experiments: dynamic mechanical analysis (DMA) as a function of temperature and electric field [Fig. 2(a)] and differential scanning calorimetry (DSC) also as a function of electric field [Fig. 2(b)].

Dynamic mechanical analysis measures the mechanical storage modulus and is very sensitive to structural phase tran- sitions [42]. Under zero field, $\mathrm{PbZrO}_{3}$ on heating shows only one sharp minimum at the antiferroelectric-paraelectric (AFEPE) phase transition, as expected, and consistent also with the dielectric constant measurements [Fig. 1(a)]. With voltage, the mechanical singularity splits into two: one that shifts to higher temperatures (consistent with a FE-PE transition) while the other moves to lower temperatures with increasing field (consistent with an AFE-FE transition).

It is interesting to notice that the field-induced polar phase can indeed be stable (ferroelectriclike), as shown by the ferroelectric hysteresis loops [inset of Fig. 1(b)]. Even in the absence of external field, this ferroelectric phase can appear on cooling [43-47], and is responsible for the second anomaly of the dielectric constant [Fig. 1(a)]; on heating, however, the ferroelectric phase only appears when a high enough energetic external stimulus, such as a voltage, is applied to the system. Therefore, if the polar phase is unstable, removal of the field returns the material to its antipolar phase, yielding the typical antiferroelectric double-hysteresis loop. If it is stable, however, the material stays "locked" into a ferroelectric state even after the field is removed, so subsequent voltage pulses do not modify the polar state, yielding a standard FE hysteresis loop. This behavior translates into different electrocaloric responses.

DSC measurements in Fig. 2(b) show the heat flow $d Q / d T$ of $\mathrm{PbZrO}_{3}$ bulk ceramic at four different electric fields as a function of temperature, both on heating and on cooling. A single endothermic peak on heating is observed at $0 \mathrm{kV} \mathrm{cm}^{-1}$ [black curve in Fig. 2(b)]. This peak corresponds to an endothermic AFE-PE first-order transition (latent heat), and is consistent both with the dielectric [Fig. 1(a)] and electromechanical results [Fig. 2(a)]. Like the DMA, the DSC also shows this splitting into two distinct peaks with the increasing electric field. The lower-temperature one, which corresponds to the AFE-FE phase transition, shifts towards ever-lower temperatures with increasing field. The second peak (FE-PE) 


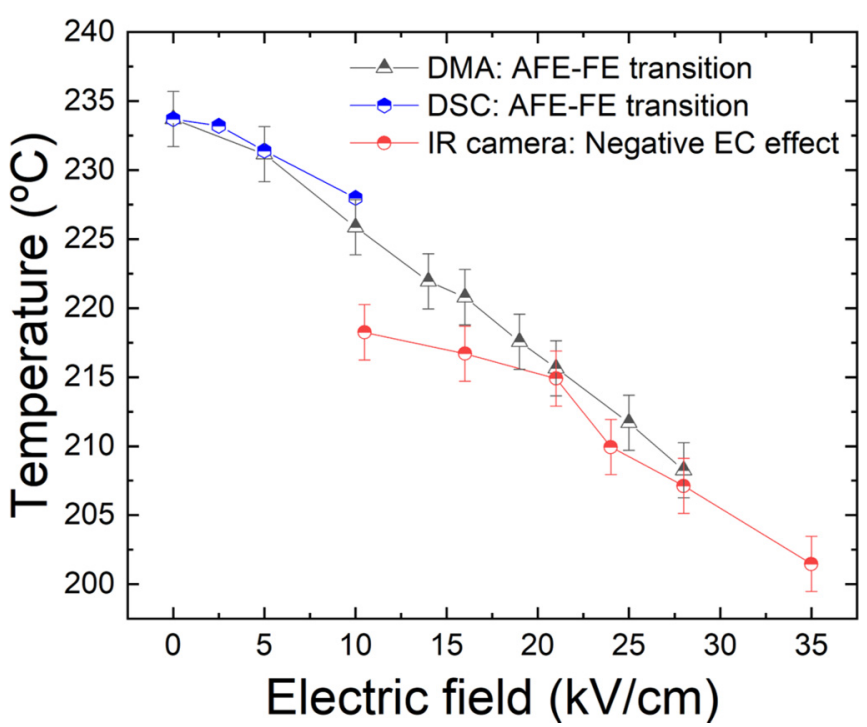

FIG. 3. Critical temperature of the AFE-FE phase transition as a function of field, measured by DMA (black) and DSC (blue), and onset temperature of giant negative electrocaloric effect measured by the infrared camera. The three independent measurements coincide.

moves towards high temperatures. Therefore, applying an electric field on our bulk $\mathrm{PbZrO}_{3}$ stabilizes the ferroelectric phase (Supplemental Material [8], Fig. S10). This behavior is analogous to that of DMA [Fig. 2(a)].

\section{DISCUSSION}

Based on the presented results, we can now draw a conclusion on the origin of the large electrocaloric effect. Qualitatively, we observe that the transitions between weak (region 1) and large responses (region 2) are abrupt [Fig. 1(b)], and the AFE and FE hysteresis loops [inset in Fig. 1(b)] ex- ceed the phase-transition field $E_{\mathrm{AFE}-\mathrm{FE}}$ and display saturation. These observations, together with DSC data, thus provide definitive evidence for the link between "giant" electrocaloric effects (both negative and positive) and field-induced phase transitions: as displayed by the DSC data [Fig. 2(b)] the AFEFE peak corresponds to an endothermic transition, which yields cooling upon field application, i.e., a negative electrocaloric effect [region 2 in Fig. 1(b)]. In fact, the large negative electrocaloric effect in region 2 starts precisely at the phase-transition temperatures given by DMA and DSC, as shown in Fig. 3. Conversely, the PE-FE peak is exothermic and thus linked to a positive electrocaloric effect [region 4 in Fig. 1(a)].

All evidence thus indicates that even if there is some dipole canting contribution [8] [range 1 in Fig. 1(b)] above the AFE-FE transition point $\left(E<E_{\mathrm{AFE}-\mathrm{FE}}\right)$, its role is negligible compared to that of the latent heat of transformation. In addition, our maximum negative $\Delta T$ matches atomistic calculations for the antiferroelectric-ferroelectric phase boundary [48], which is also consistent with a first-order phasetransition origin.

Based on the different measurements, it is possible to draw a temperature-field phase diagram for $\mathrm{PbZrO}_{3}$ [Fig. 4(a)] including the three phases: antiferroelectric, ferroelectric, and paraelectric. Based on this phase diagram, we have also generated a thermodynamic scheme showing how the electrocaloric effect works in the different regimes [Fig. 4(b) and Fig. S7(b)]; this scheme should be valid for any antiferroelectric with an endothermic AFE-FE phase transition:

(1) Region 1 corresponds to a dipole canting response where no phase transition takes place. Thus, we have a reversible system where the negative $\Delta T$ achieved when the voltage is turned on $\left(V_{\mathrm{ON}}\right)$ reverses into positive $\Delta T$ of similar magnitude when it is turned off $\left(V_{\mathrm{OFF}}\right)$.

(2) Region 2 is linked to the endothermic AFE-FE phase transition [Fig. 2(b)]. Notice that the expected field-

(a)

(b)

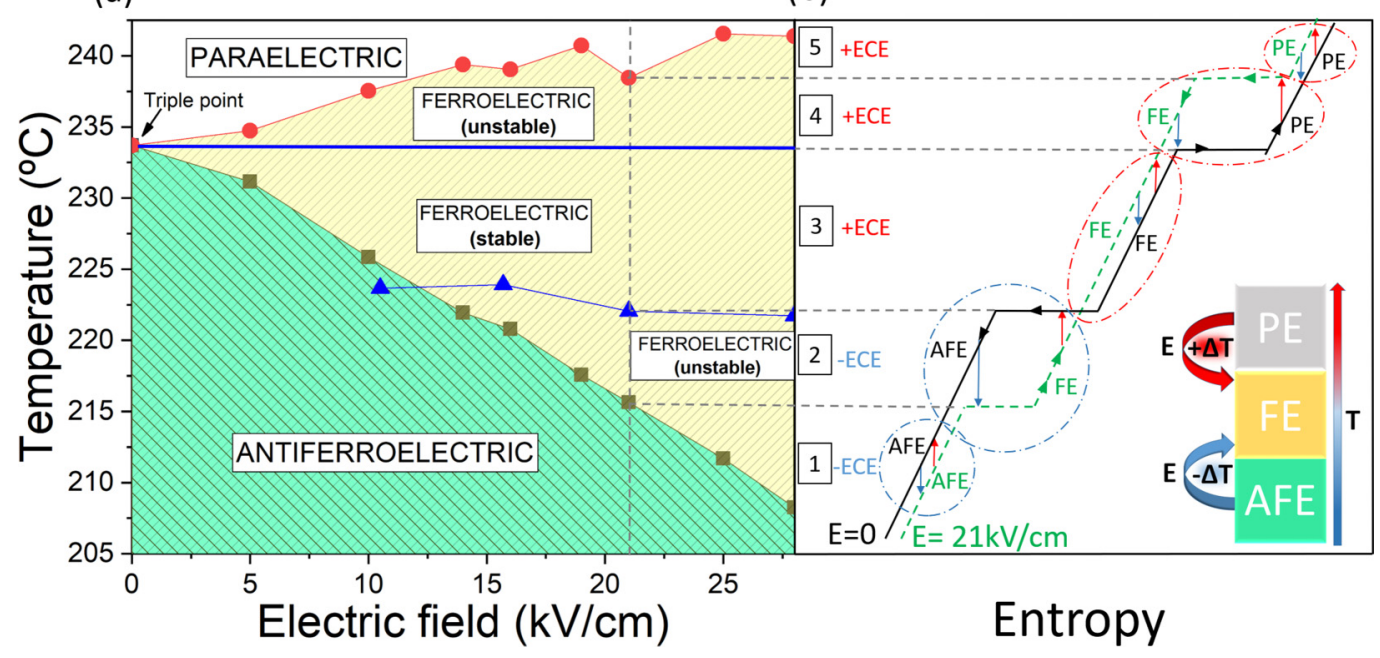

FIG. 4. (a) Tentative phase diagram for $\mathrm{PbZrO}_{3}$ as a function of electric field. AFE-FE and FE-PE transitions are plotted with electromechanical results (DMA) while the ferroelectric/field-induced ferroelectric transition is plotted with electrocaloric data (IR camera). (b) Schematic $T-S$ diagram of $\mathrm{PbZrO}_{3}$ showing all phase transitions for $E=0$ and $E=21 \mathrm{kV} / \mathrm{cm}$. The arrows represent the adiabatic $(\Delta S=0)$ electrocaloric $\Delta T$ when an electric field $E$ is applied or removed. All the different electrocaloric scenarios are labeled as in Fig. 1(b). The inset in (b) represents the connection between the phase transitions in $\mathrm{PbZrO}_{3}$ and its positive and negative electrocaloric responses. 
induced temperature changes are not symmetric, and this has been experimentally confirmed (Supplemental Material [8], Fig. S7(a) and Fig. S9(b)].

(3) In region 3 the $\mathrm{FE}$ phase is stable, and thus a regular positive ECE takes place, with a symmetric $V_{\mathrm{ON}} / V_{\mathrm{OFF}}$ response [Figs. S1-S6 and Fig. S8(e)].

(4) Region 4 corresponds to the PE-FE phase transition upon field application, that is, the large positive ECE response typical of ferroelectrics close to $T_{C}$.

(5) Region 5 yields a low positive ECE following the same standard electrocaloric mechanism as in region 3: slight increase in dipole alignment yields slight changes in temperature.

Therefore, we have answered the basic question about the origin of the so-called giant negative ECE in antiferroelectric $\mathrm{PbZrO}_{3}$ : this large response $(\Delta T=-3.5 \mathrm{~K})$ is a latent-heat mediated ECE coming from the first-order AFE-FE phase transition. In contrast, smaller negative ECE responses $(\Delta T \lesssim$ $-0.6 \mathrm{~K}$ ) have its origin in the dipolar destabilization (canting) model.

Our results for pure $\mathrm{PbZrO}_{3}$ are opposite to those measured in La-doped $\mathrm{Pb}(\mathrm{Zr}, \mathrm{Ti}) \mathrm{O}_{3}$ (PLZT) [49,50] or $\mathrm{Pb}_{0.99} \mathrm{Nb}_{0.02}\left[\left(\mathrm{Zr}_{0.58} \mathrm{Sn}_{0.43}\right)_{0.92} \mathrm{Ti}_{0.08}\right]_{0.98} \mathrm{O}_{3}$ (PNZST) antiferroelectrics [41] which display a positive electrocaloric effect when the AFE-FE transition takes place $\left(E<E_{\mathrm{AFE}-\mathrm{FE}}\right)$. The difference seems to be correlated with the doping-induced change in the position of the FE phase relative to the AFE one in the material's phase diagram [51], where a negative ECE by indirect methods was achieved in an AFE-FE phase sequence in PNZST 13/2/2. While the low-temperature phase (i.e., the ground state) is antiferroelectric for $\mathrm{PbZrO}_{3}$ and $\mathrm{Ba}$-doped $\mathrm{PbZrO}_{3}$ [23], it is ferroelectric for PLZT and PNZST. Hence, the polar phase has higher entropy and therefore the field-induced transition to the polar phase is endothermic for $\mathrm{PbZrO}_{3}$, while the opposite is true for PLZT and PNZST. In fact, first-principle calculations also on the archetype antiferroelectric $\mathrm{PbZrO}_{3}$ [52], but in this case with a FE-AFE-PE phase sequence i.e FE phase as the ground state, show that the AFE-FE phase transition yields a positive electrocaloric effect, contrary to our negative response.

Our direct measurements also show a very large positive electrocaloric peak $(\Delta T=+5.6 \mathrm{~K})$ in $\mathrm{PbZrO}_{3}$, linked to the transition between the paraelectric phase and the intermediate ferroelectric phase. The link between the giant negative response and antiferroelectric switching not only affects the field range in which the response is maximized, but also has functional consequences for the useful temperature range. As long as the external field is bigger than the antiferroelectricferroelectric phase-transition field $E_{\mathrm{AFE}-\mathrm{FE}}$ and smaller than the breakdown field, there will be switching and hence a large negative ECE. This is in contrast to the equivalent positive ECE peak of ferroelectrics, which is tied to their field-induced paraelectric-ferroelectric transition and thus anchored to their Curie temperature. In our $\mathrm{PbZrO}_{3}$ ceramics, the maximum applied field was $E=42 \mathrm{kV} \mathrm{cm}^{-1}$ [Fig. 1(b)], sufficient to cause switching and concomitant large ECE over a range of $\sim 20 \mathrm{~K}$ below the Curie temperature, but in thin films it is possible to achieve antiferroelectric switching even at room temperature [53]. Since the absolute value of the ECE is directly proportional to the absolute temperature of the sample [Eq. (1)], a reduction in the ECE is still expected at room temperature, proportional to the ratio between room temperature and Curie temperature, which is about $60 \%$ for the specific case of $\mathrm{PbZrO}_{3}$. Nevertheless, this is still a substantial negative ECE that can in theory be retained over a temperature range $200^{\circ}$ below the Curie point. The link to antiferroelectric switching thus potentially gives the negative electrocaloric effect a practical advantage over the positive effect of ferroelectrics in terms of wide temperature range of application.

\section{SUMMARY}

The present work shows that the large electrocaloric effect of antiferroelectric $\mathrm{PbZrO}_{3}$ does not arise from a continuous destabilization of the antiparallel sublattice, but from the field-induced first-order endothermal transition between the antiferroelectric and ferroelectric phases. This has important implications for the functional response of the material: it means that the effect does not disappear above coercive fields, it links the dynamics of the electrocaloric response to the switching dynamics of the antiferroelectrics, and it implies that large negative electrocaloric effects can in principle be achieved over the entire field-temperature range of the AFEFE transition.

\section{MATERIALS AND EXPERIMENTAL METHODS}

\section{A. Fabrication and sample preparation}

We have measured the electrocaloric effect of ceramics of the perovskite antiferroelectric archetype, $\mathrm{PbZrO}_{3}$. The samples were made as described in Ref. [20] and disk polished down to thicknesses between 100 and $150 \mu \mathrm{m}$ with a Multiprep polishing system. Platinum electrodes were deposited by electron beam evaporation and platinum wires bonded with curated silver paste.

\section{B. Dielectric measurements}

The capacitance and losses were measured (Agilent Precision LCR Meter, model E-4980A) as a function of temperature to establish their quality (low losses) and pinpoint the antiferroelectric-paraelectric phase transition. Polar hysteresis loops were measured with a Radiant LC meter at $1 \mathrm{kHz}$ in order to establish the antiferroelectric/ferroelectric/dielectric nature of the different phases. The samples' temperature was controlled in a Linkam system in vacuum to increase the air breakdown field.

\section{Dynamic mechanical analysis}

Mechanical properties were done using a Perkin-Elmer Dynamic Mechanical Analyzer model 8, equipped with an in situ oven in a three-point bending geometry. Samples were connected to an external high-voltage source while mechanically stressed between 1-3 Hz. To improve mechanical robustness, the samples used for mechanical analysis were thicker than those used for electrocaloric measurements, between 250 and $350 \mu \mathrm{m}$ in thickness. The electric field was applied continuously to the sample as it heated up and cooled 
down. The error in absolute temperature of the samples (discrepancies between the thermocouples used in the different experimental setups) is $\leqslant 2 \mathrm{~K}$.

\section{Infrared electrocaloric characterization}

The electrocaloric performance was measured by infrared (IR) thermometry, using two different infrared cameras to ratify the robustness of the result. The cameras were an FLIR x6580sc and an FLIR SC5500 with acquisition speeds (frames per second, fps) of 130 and $376 \mathrm{fps}$, respectively, and a field of view of $3.2 \mathrm{~mm} \times 2.55 \mathrm{~mm}$. Prior to the IR characterization, the samples were covered with an emissivity-calibrated black paint. The temperature was controlled with a Linkam system. The electrocaloric effect was induced by voltage delivered by a Keithley High Voltage Sourcemeter 2410, with source current capped at $0.1 \mathrm{~mA}$. The measuring process is based on the dynamics of a Brayton cycle: applying a voltage step adiabatically, acquire the response and let the sample thermalize before adiabatically removing the field. The relative temperature changes acquired with the IR camera were measured with an accuracy of $0.1{ }^{\circ} \mathrm{C}$.

\section{E. Differential scanning calorimetry}

Using a commercial DSC, NEZTSCH, heat flux $d Q / d t$ measurements at zero field and under three electric fields (2.5,
$5,10 \mathrm{kV} \mathrm{cm}^{-1}$ ) were done on 6.00-mg bulk ceramic $\mathrm{PbZrO}_{3}$ at a heating rate of $10 \mathrm{~K} \mathrm{~min}^{-1}$. The electric field was maintained fixed in the material during the measurement. From heat-flux measurements and after removal of the baseline we compute the heat-flow measurements $d Q / d T$ shown in Fig. 2(b).

\section{ACKNOWLEDGMENTS}

We acknowledge financial support to ICN2, which is funded by the CERCA programme/Generalitat de Catalunya and by the Severo Ochoa programme of the Spanish Ministry of Economy, Industry and Competitiveness (MINECO, Grant No. SEV-2017-0706). We also acknowledge support to Plan Nacional (MINECO Grant No. MAT2016-77100-C2-1-P) and MINECO Grant No. BES-2016-077392. R.F., Y.N., and E.D acknowledge the Luxembourg National Research Fund (FNR) for funding part of this research through the Projects No. CAMELHEAT/C17/MS/11703691/Defay and No. COFERMAT/P12/4853155/Kreisel. This work was also supported in part by the Spanish Ministry of Science, Innovation and Universities under the HIPERCELLS Project No. RTI2018098392-B-I00, the Regional Government of the Generalitat de Catalunya under Grants No. 2017 SGR 1384 and No. 217 SGR 1384, the Consejo Superior de Investigaciones Científicas through internal research programs with Identification No. 2018501063 and No. 201950E036. This work was also supported by the National Science Centre, Poland, within the Project No. 2016/21/B/ST3/02242.
[1] Q. Correia and T. Zhang, Electrocaloric Materials: New Generation of Coolers (Springer, Berlin, 2014).

[2] W. Thomson, On the thermoelastic, thermomagnetic and pyroelectric properties of matter, Philos. Mag. 5, 4 (1878).

[3] J. Kobeko and P. Kurtschatov, Dielectrische eigenshaften der seignettesalykristalle, Z. Phys. 66, 192 (1930).

[4] J. F. Hautzenlaub, Electric and dielectric behavior of potassium dihydrogen phosphate, Ph.D. thesis, Massachusetts Institute of Technology, 1943.

[5] A. Mischenko, Q. Zhang, J. F. Scott, R. W. Whatmore, and N. D. Mathur, Giant electrocaloric effect in thin film $\mathrm{Pb} \mathrm{Zr} 0.95 \mathrm{Ti}_{0.05}$ $\mathrm{O}_{3}$, Science 311, 1270 (2006).

[6] N. Kaminski, The ideal chip is not enough: Issues retarding the success of wide band-gap devices, Jpn. J. Appl. Phys. 56, 04CA03 (2017).

[7] Y. Bai, G. P. Zheng, and S. Q. Shi, Abnormal electrocaloric effect of $\mathrm{Na}_{0.5} \mathrm{Bi}_{0.5} \mathrm{TiO}_{3}-\mathrm{BaTiO}_{3}$ lead-free ferroelectric ceramics above room temperature, Mater. Res. Bull. 46, 1866 (2011).

[8] See Supplemental Material at http://link.aps.org/supplemental/ 10.1103/PhysRevB.103.054112 for additional information on the electrocaloric, mechanical and calorimetric properties of $\mathrm{PbZrO}_{3}$ ceramics.

[9] F. Le Goupil, A. Berenov, A.-K. Axelsson, M. Valant, and N. M. Alford, Direct and indirect electrocaloric measurements on $\langle 001\rangle-\mathrm{PbMg}_{1 / 3} \mathrm{Nb}_{2 / 3} \mathrm{O}_{3}-30 \mathrm{PbTiO}_{3}$ single crystals, J. Appl. Phys. 111, 124109 (2012).
[10] Y. Liu, J. F. Scott, and B. Dkhil, Direct and indirect measurements on electrocaloric effect: Recent developments and perspectives, Appl. Phys. Rev. 3, 031102 (2016).

[11] X. Li, S. G. Lu, X. Z. Chen, H. Gu, X. S. Qian, and Q. M. Zhang, Pyroelectric and electrocaloric materials, J. Mater. Chem. C 1, 23 (2013).

[12] M. Marathe, A. Grünebohm, T. Nishimatsu, P. Entel, and C. Ederer, First-principles-based calculation of the electrocaloric effect in $\mathrm{BaTiO}_{3}$ : A comparison of direct and indirect methods, Phys. Rev. B 93, 054110 (2016).

[13] S. Crossley, B. Nair, R. W. Whatmore, X. Moya, and N. D. Mathur, Electrocaloric Cooling Cycles in Lead Scandium Tantalate with True Regeneration via Field Variation, Phys. Rev. X 9, 041002 (2019).

[14] W. Geng, Y. Liu, X. Meng, L. Bellaiche, J. F. Scott, B. Dkhil, and A. Jiang, Giant negative electrocaloric effect in antiferroelectric La-Doped $\mathrm{Pb}(\mathrm{ZrTi}) \mathrm{O}_{3}$ thin films near room temperature, Adv. Mater. 27, 3165 (2015).

[15] I. Ponomareva and S. Lisenkov, Bridging the Macroscopic and Atomistic Descriptions of the Electrocaloric Effect, Phys. Rev. Lett. 108, 167604 (2012).

[16] J. Peräntie, J. Hagberg, A. Uusimäki, and H. Jantunen, Electricfield-induced dielectric and temperature changes in a $\langle 001\rangle$ Oriented $\mathrm{Pb}\left(\mathrm{Mg}_{1 / 3} \mathrm{Nb}_{2 / 3}\right) \mathrm{O}_{3}-\mathrm{PbTiO}_{3}$ single crystal, Phys. Rev. B 82, 134119 (2010).

[17] M. Marathe, D. Renggli, M. Sanlialp, M. O. Karabasov, V. V. Shvartsman, D. C. Lupascu, A. Grünebohm, and C. Ederer, Electrocaloric effect in $\mathrm{BaTiO}_{3}$ at all three ferroelectric 
transitions: Anisotropy and inverse caloric effects, Phys. Rev. B 96, 014102 (2017).

[18] R. Pirc, B. Rožič, J. Koruza, B. Malič, and Z. Kutnjak, Negative electrocaloric effect in antiferroelectric $\mathrm{PbZrO}_{3}, \mathrm{Epl} \mathrm{107,} 17002$ (2014).

[19] A. Grünebohm, Y. Bin Ma, M. Marathe, B. X. Xu, K. Albe, C. Kalcher, K. C. Meyer, V. V. Shvartsman, D. C. Lupascu, and C. Ederer, Origins of the inverse electrocaloric effect, Energy Technol. 6, 1491 (2018).

[20] K. Roleder and K. Wojcik, The electrostrictive effect and ferroelectric properties in $\mathrm{PbZrO}_{3}$, Ferroelectrics 61, 293 (1984).

[21] K. M. Rabe, Antiferroelectricity in oxides: A reexamination, in Functional Metal Oxides: New Science and Novel Applications (2013), pp. 221-244.

[22] X. Tan, C. Ma, J. Frederick, S. Beckman, and K. G. Webber, The antiferroelectric $\leftrightarrow$ ferroelectric phase transition in leadcontaining and lead-free perovskite ceramics, J. Am. Ceram. Soc. 94, 4091 (2011).

[23] R. Pirc, B. Rožič, J. Koruza, G. Cordoyiannis, B. Malič, and Z. Kutnjak, Anomalous dielectric and thermal properties of BaDoped $\mathrm{PbZrO}_{3}$ ceramics, J. Phys.: Condens. Matter 27, 455902 (2015).

[24] J. Li, S. Qin, Y. Bai, J. Li, and L. Qiao, Flexible control of positive and negative electrocaloric effects under multiple fields for a giant improvement of cooling capacity, Appl. Phys. Lett. 111, 093901 (2017).

[25] M. Wu, Q. Zhu, J. Li, D. Song, H. Wu, M. Guo, J. Gao, Y. Bai, Y. Feng, S. J. Pennycook, and X. Lou, Electrocaloric effect in ferroelectric ceramics with point defects, Appl. Phys. Lett. 114,142901 (2019).

[26] P. Z. Ge, X. D. Jian, X. W. Lin, X. G. Tang, Z. Zhu, Q. X. Liu, Y. P. Jiang, T. F. Zhang, and S. G. Lu, Composition dependence of giant electrocaloric effect in $\mathrm{Pb}_{x} \mathrm{Sr}_{1-X} \mathrm{TiO}_{3}$ ceramics for energyrelated applications, J. Mater. 5, 118 (2019).

[27] X. D. Jian, B. Lu, D. D. Li, Y. B. Yao, T. Tao, B. Liang, J. H. Guo, Y. J. Zeng, J. Le Chen, and S. G. Lu, Direct measurement of large electrocaloric effect in $\mathrm{Ba}\left(\mathrm{Zr}_{x} \mathrm{Ti}_{1-x}\right) \mathrm{O}_{3}$ ceramics, ACS Appl. Mater. Interfaces 10, 4801 (2018).

[28] S. Lu, G. Chen, Y. Zhang, Z. Zhao, F. Li, Z. Lv, Z. Ma, D. Wang, C. Lu, and S. Li, Electrocaloric Effect in Lead-Free $0.5 \mathrm{Ba}\left(\mathrm{Zr}_{0.2} \mathrm{Ti}_{0.8}\right) \mathrm{O}_{3}-0.5\left(\mathrm{Ba}_{0.7} \mathrm{Ca}_{0.3}\right) \mathrm{TiO}_{3}$ Ceramic Measured by Direct and Indirect Methods, Ceram. Int. 44, 21950 (2018).

[29] N. Goupil, F. Le, A. Baker, F. Tonus, A. Berenov, A. Randall, Clive, and M. Alford, Direct measurement of electrocaloric effect in lead-free $\left(\mathrm{Na}_{0.5} \mathrm{Bi}_{0.5}\right) \mathrm{TiO}_{3}$-based multilayer ceramic capacitors, J. Eur. Ceram. Soc. 39, 3315 (2013).

[30] Q. Wei, M. Zhu, M. Zheng, Y. Hou, J. Li, and Y. Bai, Large electrocaloric effect near room temperature in lead-free scanning calorimetry, Scr. Mater. 171, 10 (2019).

[31] H. Zaitouni, L. Hajji, D. Mezzane, E. Choukri, A. Alimoussa, S. Ben Moumen, B. Rožič, M. E. Marssi, and Z. Kutnjak, Direct electrocaloric, structural, dielectric, and electric properties of lead-free ferroelectric material $\mathrm{Ba}_{0.9} \mathrm{Sr}_{0.1} \mathrm{Ti}_{1-X} \mathrm{SnxO}_{3}$ synthesized by semi-wet method, Phys. B: Condens. Matter 566, 55 (2019).

[32] T. Li, X. Liu, S. Shi, Y. Yin, H. Li, Q. Wang, Y. Zhang, J. Bian, S. S. Rajput, C. Long, B. Peng, Y. Bai, Y. Wang, and X. Lou, Large electrocaloric efficiency over a broad temperature span in lead-free $\mathrm{BaTiO}_{3}$-based ceramics near room temperature, Appl. Phys. Lett. 111,202902 (2017).
[33] A. K. Axelsson, F. L. Goupil, M. Valant, and N. M. N. Alford, Optimisation of $\mathrm{SrBi}_{2}(\mathrm{Nb}, \mathrm{Ta})_{2} \mathrm{O}_{9}$ aurivillius phase for lead-free electrocaloric cooling, J. Eur. Ceram. Soc. 38, 5354 (2018).

[34] F. L. Goupil, A. K. Axelsson, L. J. Dunne, M. Valant, G. Manos, T. Lukasiewicz, J. Dec, A. Berenov, and N. M. N. Alford, Anisotropy of the electrocaloric effect in lead-free relaxor ferroelectrics, Adv. Energy Mater. 4, 1301688 (2014).

[35] Y. Matsushita, A. Nochida, T. Yoshimura, and N. Fujimura, Direct measurements of electrocaloric effect in ferroelectrics using thin-film thermocouples, Jpn. J. Appl. Phys. 55, 10 TB04 (2016).

[36] V. S. Bondarev, I. N. Flerov, M. V. Gorev, E. I. Pogoreltsev, M. S. Molokeev, E. A. Mikhaleva, A. V. Shabanov, and A. V. Es'kov, Influence of thermal conditions on the electrocaloric effect in a multilayer capacitor based on doped $\mathrm{BaTiO}_{3}$, J. Adv. Dielectr. 7, 1750041 (2017).

[37] Y. Bai, K. Ding, G. P. Zheng, S. Q. Shi, and L. Qiao, Entropychange measurement of electrocaloric effect of $\mathrm{BaTiO}_{3}$ single crystal, Phys. Status Solidi A 209, 941 (2012).

[38] G. Chen, Y. Zhang, X. Chu, G. Zhao, F. Li, J. Zhai, Q. Ren, B. Li, and S. Li, Large electrocaloric effect in Ladoped $0.88 \mathrm{~Pb}\left(\mathrm{Mg}_{1 / 3} \mathrm{Nb}_{2 / 3}\right) \mathrm{O}_{3}-0.12 \mathrm{PbTiO}_{3}$ relaxor ferroelectric ceramics, J. Alloys Compd. 727, 785 (2017).

[39] S. Crossley, T. Usui, B. Nair, S. Kar-Narayan, X. Moya, S. Hirose, A. Ando, and N. D. Mathur, Direct electrocaloric measurement of $0.9 \mathrm{~Pb}\left(\mathrm{Mg}_{1 / 3} \mathrm{Nb}_{2 / 3}\right) \mathrm{O}_{3}-0.1 \mathrm{PbTiO}_{3}$ films using scanning thermal microscopy, Appl. Phys. Lett. 108, 032902 (2016).

[40] B. Nair, T. Usui, S. Crossley, S. Kurdi, G. G. Guzmán-Verri, X. Moya, S. Hirose, and N. D. Mathur, Large electrocaloric effects in oxide multilayer capacitors over a wide temperature range, Nature (London) 575, 468 (2019).

[41] N. Novak, F. Weyland, S. Patel, H. Guo, X. Tan, J. Rödel, and J. Koruza, Interplay of conventional with inverse electrocaloric response in $(\mathrm{Pb}, \mathrm{Nb})(\mathrm{Zr}, \mathrm{Sn}, \mathrm{Ti}) \mathrm{O}_{3}$ antiferroelectric materials, Phys. Rev. B 97, 094113 (2018).

[42] W. Schranz, Dynamic mechanical analysis-a powerful tool for the study of phase transitions, Phase Transitions 64, 103 (1997).

[43] H. Liu, Origin of the intermediate phase in lead zirconate, $\mathrm{PbZrO}_{3}$, J. Am. Ceram. Soc. 101, 5281 (2018).

[44] G. Shirane, Ferroelectricity and antiferroelectricity in ceramic $\mathrm{PbZrO}_{3}$ containing Ba or Sr, Phys. Rev. 86, 219 (1952).

[45] E. Sawaguchi, Ferroelectricity versus antiferroelectricity in the solid solutions of $\mathrm{PbZrO}_{3}$ and $\mathrm{PbTiO}_{3}$, J. Phys. Soc. Jpn. 8, 615 (1953).

[46] B. A. Scott and G. Burns, Crystal growth and observation of the ferroelectric phase of $\mathrm{PbZrO}_{3}$, J. Am. Ceram. Soc. 55, 331 (1972).

[47] A. Bussmann-Holder, J. H. Ko, A. Majchrowski, M. Górny, and K. Roleder, Precursor dynamics, incipient ferroelectricity and huge anharmonicity in antiferroelectric lead zirconate $\mathrm{PbZrO}_{3}$, J. Phys.: Condens. Matter 25, 212202 (2013).

[48] A. V. Kimmel, O. T. Gindele, D. M. Duffy, and R. E. Cohen, Giant electrocaloric effect at the antiferroelectric-to-ferroelectric phase boundary in $\mathrm{Pb}\left(\mathrm{Zr}_{x} \mathrm{Ti}_{1-x}\right) \mathrm{O}_{3}$, Appl. Phys. Lett. 115, 023902 (2019).

[49] A. Peláiz-Barranco, J. Wang, and T. Yang, Direct and indirect analysis of the electrocaloric effect for lanthanum-modified lead zirconate titanate antiferroelectric ceramics, Ceram. Int. 42, 229 (2016). 
[50] B. Lu, P. Li, Z. Tang, Y. Yao, X. Gao, W. Kleemann, and S. G. $\mathrm{Lu}$, Large electrocaloric effect in relaxor ferroelectric and antiferroelectric lanthanum doped lead zirconate titanate ceramics, Sci. Rep. 7, 45335 (2017).

[51] Z. Xu, Z. Fan, X. Liu, and X. Tan, Impact of phase transition sequence on the electrocaloric effect in $\mathrm{Pb}(\mathrm{Nb}, \mathrm{Zr}, \mathrm{Sn}, \mathrm{Ti}) \mathrm{O}_{3}$ ceramics, Appl. Phys. Lett. 110, 082901 (2017).
[52] E. Glazkova-Swedberg, J. Cuozzo, S. Lisenkov, and I. Ponomareva, Electrocaloric effect in $\mathrm{PbZrO}_{3}$ thin films with antiferroelectric-ferroelectric phase competition, Comput. Mater. Sci. 129, 44 (2017).

[53] A. Pérez-Tomás, M. Lira-Cantú, and G. Catalan, Abovebandgap photovoltages in antiferroelectrics, Adv. Mater. 28, 9644 (2016). 